\title{
Direct Observation of Conformational Change of c-Cbl Ubiquitin Ligase by High Speed Atomic Force Microscopy
}

\author{
Yoshiki Takahashi $^{1}$, Jun-ichi Kishikawa ${ }^{1,2}$, Hiromi Tanaka ${ }^{1}$, Hiroki Konno ${ }^{1}$ \\ 1. Bio-AFM Frontier Research Center, Kanazawa University, Kanazawa, Japan. \\ 2. Fac. Life Sci., Kyoto Sangyo University, Kyoto, Japan.
}

Ubiquitination is a post-translational modification that regulates cellular process such as proteasomal degradation, DNA repair, and signal transduction pathway. Ubiquitination of substrate protein occur by transfer of ubiquitin via E1, E2 and E3. The c-Cbl belongs to a family of RING-type ubiquitin ligase E3. Physiological role of $\mathrm{c}-\mathrm{Cbl}$ is to add ubiquitin to EGFR tyrosine kinase, and the kinase containing ubiquitin chain is finally degraded by $26 \mathrm{~S}$ proteasome. Thus, through ubiquitin system, the activity of EGFR tyrosine kinase is negatively regulated in the cell. It is also known that large conformational change of c-Cbl is induced by phosphorylation, and it significantly enhance the ubiquitination activity of $\mathrm{c}-\mathrm{Cbl}$ (c-Cbl active form). The relative positioning of the linker region (LHR) and the RING domain in the tyrosine kinase binding domain (TKB domain) of phosphorylated c-Cbl is much different from that of non-phosphorylated c-Cbl [1]. To understand the dynamics of structural change during phosphorylation and following ubiquitination of substrate protein in real time, we first prepared the cCbl mutatnt (Y368E/Y371E), which mimics the phosphorylated c-Cbl [2], and compared its structural feature to wild type c-Cbl by using High-speed atomic force microscopy (HS-AFM). HS-AFM recently emerged enables direct imaging of single proteins in a physiological solution at a rate of 5-20 frames per second $[3,4]$.

To evaluate active (phosphorylated) and inactive (non-phosphorylated) forms of c-Cbl, we first examined self-ubiquitination of c-Cbl. In addition to the activity of ubiquitin transfer to substrate protein by c-Cbl, it is known that c-Cbl itself is ubiquitinated (self-ubiquitination) and this self-ubiquitination is also enhanced by phosphorylation or Y368E, Y371E mutation [2]. Active c-Cbl shows higher selfubiquitination activity than inactive c-Cbl.

The HS-AFM was equipped with small cantilevers (BL-AC10-DS, Olympus: spring constant, $k=\sim 0.1$ $\mathrm{N} / \mathrm{m}$, resonance frequency, $f=400-500 \mathrm{kHz}$ in water) and operated in tapping mode[ando et al]. Amorphous carbon tip was grown on the top of the cantilevers by electron-beam deposition using a field emission scanning electron microscope (ELS7500, Elionix). The free oscillation amplitude during observation was $\sim 1.5 \mathrm{~nm}$ and the set-point amplitude was $80-90 \%$ of the free amplitude. Imaging rate, scan size, and feedback control were optimized during observation to enable the possible visualization with minimum tip force. Recorded images were analyzed by custom software, Kodec, prepared by N. Kodera.

By using HS-AFM, we could observed structural dynamics of RING domain on c-Cbl in real time. Active and inactive RING domain of c-Cbl showed different distributions relative to the TKB domain. Thus, phosphorylation of Y368, Y371 causes the conformational change of c-Cbl and it changes the trajectory of RING domain fluctuation and enhances ubiquitination activity of c-Cbl. 


\section{References:}

[1] H. Dou et al. Nat. Struct. Mol. Biol 19 (2012), p. 184.

[2] C.K. Kassenbrock et al. J. Biol. Chem. 279 (2004), p. 28017.

[3] T. Ando et al. Proc Natl Acad Sci USA 98 (2001), p.12468.

[4] T. Uchihashi et al Nat Protoc 7 (2012), p. 1193.
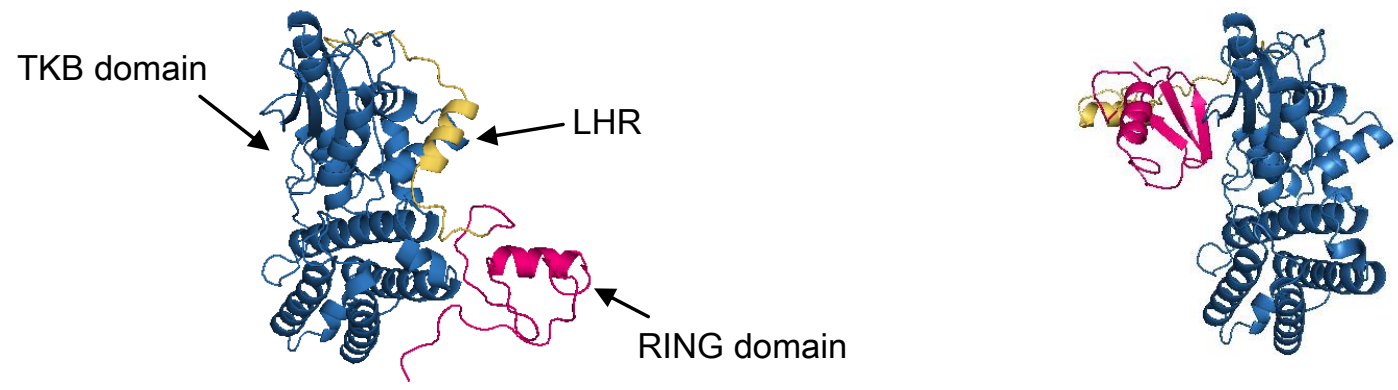

None-phosphorylated c-Cbl (Inactive)

Phosphorylated c-Cbl (Active)

PDB:1FBV

PDB:4A4B

Figure 1. Comparison of structure between none-phosphorylated c-Cbl and phosphorylated c-Cbl.
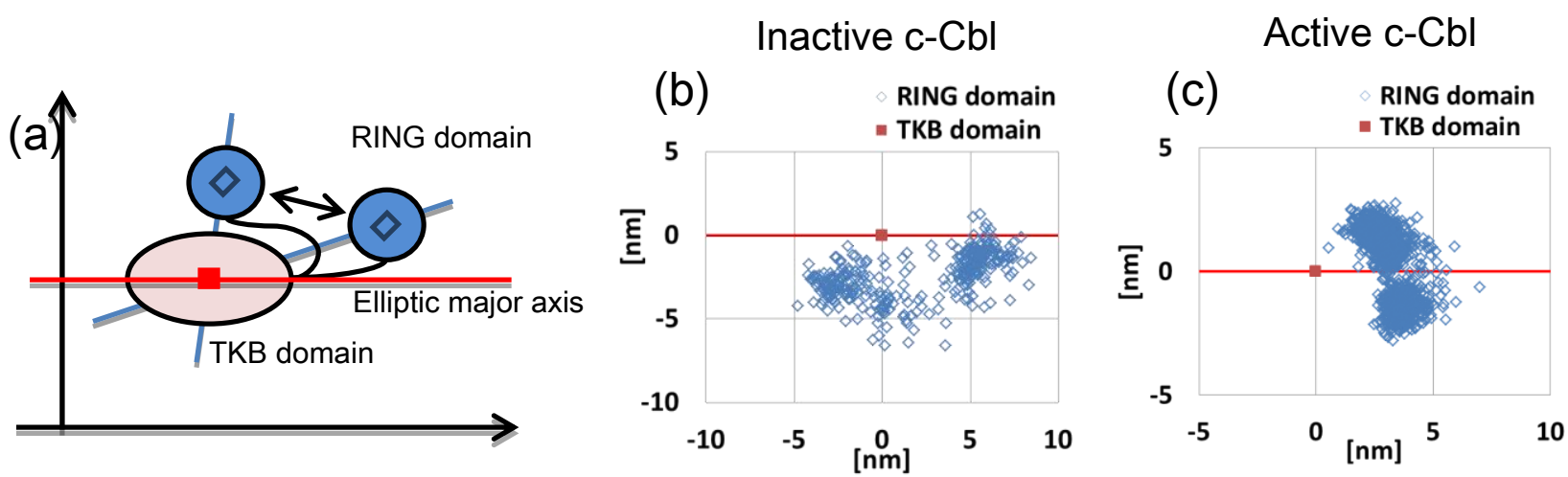

Figure 2. RING domain distribution relative to TKB central coordinate.

(a)Schematic model of analysis of RING domain distribution relative to TKB domain. Elliptic major axis along with TKB central coordinate, which is fitted to X-axis, shown as red line. Distributions of the RING domain relative to the TKB domain major axis in inactive $\mathrm{c}-\mathrm{Cbl}$ (b) and active c-Cbl (c). 